\title{
A Foundational Science Department's Transition to an Online Community
}

\author{
Kara Sawarynski $^{1}$ (1) Douglas J. Gould ${ }^{1}$ \\ Accepted: 2 September 2020 / Published online: 9 September 2020 \\ (C) International Association of Medical Science Educators 2020
}

\begin{abstract}
Like many medical school department's around the world, we needed to pivot, almost instantly to an online community. As a large and diverse foundational science department, grounded in a culture of collegiality and collaboration, we faced a host of challenges beyond immediate remote teaching. Of paramount concern to departmental leadership was - how do we maintain our culture while working remotely?
\end{abstract}

Keywords Remote teaching $\cdot$ Online learning $\cdot$ Medical Education $\cdot$ Communication $\cdot$ Technology

\section{Background}

Oakland University William Beaumont School of Medicine (OUWB) was founded on the premise that outstanding physicians are developed by emphasizing holistic physician development grounded in evidence-based medical science. As such, a culture of caring, empathy, communication, collaboration, and collegiality is of paramount importance at OUWB. This culture permeates all aspects of OUWB - starting with student, faculty, and staff recruitment, through graduation and beyond. The Department of Foundational Medical Studies (FMS) faculty at OUWB form the backbone of the school and have a key role in maintaining and supporting the culture; one of the primary ways such a culture is maintained is through consistent, transparent, and honest communication. As such, our necessary responses to the pandemic (social distancing, abiding by stay-at-home orders, and mandatory online teaching and interaction) posed a threat to the fabric of the school.

FMS is composed of 40 multi-disciplinary basic and social scientists and supporting staff and is responsible for the delivery of the majority of the foundational medical topics to our

Kara Sawarynski

sawaryns@ oakland.edu

1 Oakland University William Beaumont School of Medicine, Oakland University, Rochester, MI, USA medical students. In order to best serve our mission and students in the "new normal," our department needed to address several considerations quickly including virtual support for collaborative scholarship, administrative duties, and teaching. Administrative duties and support for scholarship that needed to be maintained included the following: moving forward with planned promotion and tenure processes, progressing open job searches, and working to maintain collaboration and communication, the latter not only for productivity sake but also for morale and culture sake.

\section{Activity}

Departmental administration recognized the need to move quickly in the short transition period and began by identifying and prioritizing achievable steps:

1. Solicit IT support for software and computer update needs to ensure access to necessary resources available to work remotely;

2. Curate already available technology options, including:

(a) Learning management system;

(b) Video conferencing and lecture recording tools

3. Create cogent "how-to" guides for less frequently used available software tools;

4. Schedule meetings with course teams, IT, and medical education administration to plan for the possibility of extended remote learning. 
As information was made available to the departmental administration, transparent and timely communication with department members was used to disseminate information regarding how the rapidly developing pandemic situation would impact their work life. Faculty were permitted to take computers and peripherals home, and were encouraged to download previously recorded lectures for use in developing online content. As faculty members began to balance work/life issues (with exposures and risk factors necessitating early self-isolation, and K-12 schools ceasing face-to-face interactions), weekly work plans with intended outcomes were developed with individuals working from home.

Importantly, we instituted a department-wide team communication platform (Slack ${ }^{\mathrm{R}}$, www.slack.com) at the beginning of the crisis [1]. Although relatively new to the medical education arena, Slack is widely accepted as a business and engineering communication tool [2]. As Slack was new to most faculty members, we quickly to developed a "how to" document and held a in person workshop. All FMS faculty members signed up to use Slack.

Approximately 1-month into the stay home order, an anonymous online survey was distributed to all FMS faculty (deemed Non-Human Subjects Research by the Oakland University IRB). The survey sought to capture changes in work habits and efforts towards different academic areas, assess perceptions on departmental communication, evaluate the use of Slack as a communication tool, and better understand what was and was not working well while at work from home. The Qualtrics survey used both Likert scale/quantitative and open-ended questions. Twenty-two FMS faculty completed the survey (out of 34 total faculty).

\section{Results and Discussion}

Overall, the survey found that the majority of faculty felt that they are maintaining productivity from home (mean 4.1 out of 5 , SD 0.83) and that the department has done a good job of fostering communication and collaboration with their colleagues (mean 4.05 out of 5, SD 0.86). Fifty-five percent of the faculty felt their workday has stayed about the same length, with $30 \%$ having longer and $15 \%$ shorter days. Compared with a normal work schedule, $60 \%$ of the faculty felt their current schedule is more dispersed throughout the day.

Regarding Slack, prior experience of the authors allowed for "on-the-job" training of other faculty members. In just over 1 month of usage, our department has sent close to 5000 messages and created over 20 collaborative channels (separate discussion spaces for sub-groups of faculty). It is important to note messages are focused mainly on curriculum development and scholarly activity pursuits, and that no student information is being transmitted via the platform. Prior to the stay at home order, our department has created scholarly interest groups (SIG) focused on scholarly pursuits with an emphasis on outcomes. Channels were created for each SIG to encourage continued pursuit of ongoing scholarly projects. Other channels created included "Online Teaching Resources," "Medical Library," and "M1/M2 Curriculum." The majority of messages sent within the platform occur during normal business hours, but users have found the tool to be advantageous when collaborating with colleagues working on a more dispersed work day schedule. Faculty also feel that Slack has helped foster increased departmental communication (mean 3.5 out of 5, SD 1.32) and that it is a useful tool to share remote teaching and work-from-home resources (mean 3.53 out of 5, SD 1.23). Faculty comments specific to Slack have included "great to have access to the program to get connected as a community in support of our students" and "can we keep the virtual community momentum indefinitely? It is great we can interact and meet without the limitations of physical spaces!" Software analytics indicate that not all faculty have taken to incorporating the new tool into their routine - on average, 25 faculty members are active in the tool.

When asked what has been working well while working at home, the major themes included scheduling one's day, working from task lists, video conferencing tools, working collaboratively in online documents, and communicating via Slack. Faculty felt that things that were working included "Having daily goals, keeping similar working routines, keeping my eyes open for new opportunities for teaching and research and others" and "communication with colleagues and students." Faculty felt that difficulties included navigating the balance of work/home responsibilities, missing face-to-face connections, and keeping a regular schedule. Looking to the future with the potential for continued remote teaching, faculty would like to see demos/training for educational tool features that may already be available. Additionally, easy access to technology support and continued access to high-quality online software tools and computer resources will be important for success.

As we were forced to delve into working through the pandemic with little warning, proactively addressing likely issues allowed for solutions to be identified prior to the implementation of the stay-at-home order, likely contributing to the maintenance of productivity through such trying times. Seeking to capitalize on a previously established culture dependent on high-levels of communication and shared goals (including the SIGs and collaborative education missions) allowed for a continuance of these aims in an online community. Department administration has sought to touch base with individual faculty almost daily through the Slack platform, which is not typical in a face-to-face setting. This has allowed for increases in both transparency in faculty activities and a 
deeper understanding of points where future departmental work could develop.

With all of the uncertainty and change at the time, not everyone was excited about the introduction of yet another variable - a new software package. We believe that holding the in person workshop to demonstrate the tool and ensure that all faculty were set up in the platform just prior to campus closure was instrumental in gaining buy-in, i.e., some permutation of a meeting to gain collective acceptance would seem necessary. In the future, it may be useful to meet one-on-one with reluctant team members who may not have been able to attend the workshop and/or are hesitant to learn another new technology. Additionally, the authors sought to create example communications within the platform to demonstrate the benefits. For example, an individual email question regarding how to voice-record lecture slides sent via email was posted in our "Online Teaching Resources" channel and led to eight faculty members offering helpful tips. Such resources led to a meaningful dialogue of shared experiences, and live on as a searchable resource. Finally, it was crucial for us to recognize that all of our team members are experiencing the stress of the pandemic. We developed a "COVID-19 updates" channel to share information from within our own health system as well as national/scientific news. We sought to maintain a sense of collegiality, sense of humor, and sense of normalcy by creating a "Comic Relief" channel and have had a daily "welcome to work" morning message. The overall feedback from departmental members has been positive, and multiple members have requested continued usage of the new communication tools beyond the stay at home order. As medical education continues to evolve in response to COVID-19, it is clear that we will all necessarily find new ways to communicate, stay productive, and remain true to our missions.

Authors' Contributions Dr. Sawarynski and Dr. Gould contributed equally to this work.

Data Availability NA

\section{Compliance with Ethical Standards}

Conflict of Interest The authors declare that they have no conflict of interest.

Ethical Approval Oakland University \# IRB-FY2020-33, exempt — not human subject research

Consent to Participate NA

Consent for Publication NA

Code Availability NA

\section{References}

1. Slack Technologies Inc. Slack. Available at: www.slack.com. Accessed 27 Apr 2020.

2. Johnson HA. Slack. JMLA. 2018;106(1):148-51. https://doi.org/10. 5195/jmla.2018.315.

Publisher's Note Springer Nature remains neutral with regard to jurisdictional claims in published maps and institutional affiliations. 